\title{
A Constructive Treatment to Elemental Life Forms through Mathematical Philosophy
}

\author{
Susmit Bagchi
}

Citation: Bagchi, S. A Constructive Treatment to Elemental Life Forms through Mathematical Philosophy. Philosophies 2021, 6, 84. https:// doi.org/10.3390/

philosophies6040084

\section{Academic Editors:}

Gordana Dodig-Crnkovic, Marcin J. Schroeder and Rossella Lupacchini

Received: 16 August 2021

Accepted: 6 October 2021

Published: 12 October 2021

Publisher's Note: MDPI stays neutral with regard to jurisdictional claims in published maps and institutional affiliations.

Copyright: (C) 2021 by the author. Licensee MDPI, Basel, Switzerland. This article is an open access article distributed under the terms and conditions of the Creative Commons Attribution (CC BY) license (https:// creativecommons.org/licenses/by/ $4.0 /)$.
Department of Aerospace and Software Engineering (Informatics), Gyeongsang National University, Jinju 660-701, Korea; profsbagchi@gmail.com

\begin{abstract}
The quest to understand the natural and the mathematical as well as philosophical principles of dynamics of life forms are ancient in the human history of science. In ancient times, Pythagoras and Plato, and later, Copernicus and Galileo, correctly observed that the grand book of nature is written in the language of mathematics. Platonism, Aristotelian logism, neo-realism, monadism of Leibniz, Hegelian idealism and others have made efforts to understand reasons of existence of life forms in nature and the underlying principles through the lenses of philosophy and mathematics. In this paper, an approach is made to treat the similar question about nature and existential life forms in view of mathematical philosophy. The approach follows constructivism to formulate an abstract model to understand existential life forms in nature and its dynamics by selectively combining the elements of various schools of thoughts. The formalisms of predicate logic, probabilistic inference and homotopy theory of algebraic topology are employed to construct a structure in local time-scale horizon and in cosmological time-scale horizon. It aims to resolve the relative and apparent conflicts present in various thoughts in the process, and it has made an effort to establish a logically coherent interpretation.
\end{abstract}

Keywords: platonism; predicate logic; monad; homotopy; probability

\section{Introduction}

The process of axiomatization is ancient, with wide array of applications in mathematics and in philosophy to coherently establish a theory to reach out to truth. The ideal goal is to attain truth in invariant forms independent of any contextual variables. In other words, the axiomatic development of a theory or knowledge aims to establish or systematize a structural form without any ambiguity and inherent contradictions within it [1]. Euclid first developed the axiomatized geometry in Elements where he made attempts to distinctly separate the primitive and the derived [1]. It is important to note that the philosophy of formal logic of Kant enables one to study abstract mathematical objects in nature [2]. The formal logic of axiomatization is often not purely sufficient to eliminate inherent ambiguity because of the process of axiomatizations of initials prior the coherent structures being established. However, in later times, Frege successfully formulated the methods of deriving logical inferences through the axioms in mathematics, which has found suitable applications in philosophy. It should be noted that Natorp and Cassirer disagreed with the concepts proposed by Frege and Russell on the ground, nature and logical demarcation [2]. According to Cassirer the New-logic is more suitable to study mathematical concepts in nature through relational structures. The mathematical philosophy of $\mathrm{New}$-logic vindicates the Neo-Kantian theory of space and time [2].

It is often argued that philosophy must employ the axiomatic and logistic methods of constructive inquiry [3]. The reason is that the logic-based constructive systems such as mathematics enable us to gain insight to a philosophical system and natural observable systems free from internal inconsistencies. For example, Alonzo Church, as a philosopher and mathematical logician, applied the method of hypothetico-deductive-rationalism while theorizing epistemological aspects of mathematics, logic and philosophy [3]. It is important 
to note that the method of mathematical and philosophical investigations made by Church can be viewed as a Platonic realism, and sometimes his opinion about realism conflicts with Frege. Moreover, the Platonic and Aristotelian philosophies include the elements of Pythagorean doctrines [4].

\subsection{Self as Life Forms and Transitions}

In the natural or material world, the concept of the self is evident through the interactions to other elements of nature actively or passively. In other words, the recognizing existence of self is a set of spontaneous computable actions in the nature or in the material world [5]. As a natural consequence, the following question appears: What is the natural world in this context? The answer was proposed by Anaxagoras in ancient times based on the multiplicity of basic particles. According to Aristotle, the "homoiomeroi" constitutes the universe, and the natural material world can be described by relational ideas [5]. However, it is noted earlier that Platonic realism, mathematical axiomatization and Neo-realism aim to understand the true essence of natural world as a set of mathematical principles, which are foundational. According to Plato, Parmenides and Leibniz, the transitions from the mathematical world to the natural (physical or material) perceivable world happen due to the limitation of computations, and as a result, spatiotemporal forms of the perceivable physical world appear [6]. It is important to note that, in general, it is thought that pure logism is not very suitable to purely understand abstract principles of the natural world; however, philosophers often apply inductive reasoning for drawing plausible conclusions and such inductive reasoning includes elements of probability theory [7].

\subsection{Motivation}

The mathematically abstract notion of space is elegantly explained by Kant. In view of Kant, space is an outer form derived from the perceptions by life forms and space can be infinite [8]. Note that the Euclidean construction of space does not conflict with the view of Kant. The mathematical philosophy of Leibniz tried to investigate the nature of mind-body relation in a space. This results in the two directions of related thoughts. In one direction, the solipsistic monad is conceptualized to represent existential knowledge, and in another direction, the monad is not necessarily solipsistic in nature considering the interactions [9]. In the second line of thought, the causal separation from the rest of the universe is inevitable. Note that, at the foundation of mathematics, the shapes of natural world forms and space-time follow the structures constructed by axioms, logic and geometry, which are topological in nature [5]. On the other hand, the Cartesian proposal of mind-body dualism in mathematical philosophy is experimentally reconstructed and presented in [10]. The experimentation on a human life form illustrates that the multiplicity of self in the mind exists, and more importantly, it is observed that, mathematically, the existences are a set of almost continuous functions in space-time controlling body (i.e., biophysical or materialistic form) dynamics. The almost continuous functions in the varying spaces of the life forms are separated by cuts. This motivates us to investigate the abstract mathematical theory of existential life forms in space-time through the birthdeath process of a materialistic element (i.e., body as a biophysical entity) in view of mathematical philosophy.

According to Carnap, the general theory and analysis should be based on intensional isomorphism [3]. The concept of isomorphism and homeomorphisms are widely used in various domains of mathematics such as algebra and topology, to name a few. The intensional isomorphism helps in determining isomorphism between two or more than two structures or statements where the local equivalency between substructures exists and, as a result such, equivalent substructures can be replaced, maintaining the overall isomorphism. The combinations of selective ingredients of Spinoza and Leibniz become a motivating factor to constructively approach to the existential nature of life forms and associated dynamics. The monadism of Leibniz emphasizes upon the concept of mathematical representation, and this paper tries to employ such an approach. Furthermore, in this paper 
we follow the analytical method of Carnap in order to prepare a constructive structure of elemental life forms in view of mathematical philosophy. It is possible that the mixed elements of Cassirer philosophy of symbolic forms, elements of Hegelian idealism and the Platonic realism of Alonzo Church can be observed in the constructive approach.

The rest of the paper is organized as follows. The descriptions about existentialism, dualism and abstractionism are presented in Section 2. The probabilistic treatment is formulated in Section 3. Section 4 presents the homotopic analysis, and finally, Section 5 concludes the paper.

\section{Existentialism, Dualism and Abstractionism}

The three broad and generalized schools of thoughts in philosophy and mathematics in explaining the perceivable nature and space are existentialism, dualism and abstractionism. The Platonism as well as the existentialism of Husserl proposed that the external sensual world and the associated perceptions are in reduced forms through local interactions with environments. The absolute realism is in a pure state which can be understood by the time-invariant mathematical abstract principles of nature. The existence of mind-body dualism further strengthens the school of thought of Plato to understand the true nature of existence in universe through the lenses of mathematics. Moreover, the philosophies of Pythagoras, Descartes, Kant and Leibniz propel the validation of the fact that the philosophical understanding of the true nature of life needs the invaluable doctrines of abstract mathematical understanding of the working of universe as a whole. Hence, the vivid understanding about existentialism, dualism and abstractionism are necessary. These approaches take distinct directions of analyses and inferences. In this section, brief presentations are made about these diverse schools of thoughts, and similarities as well as differences are pointed out whenever possible.

\subsection{Existentialism}

The theory of existentialism is clearly explained by Husserl in Lebenswelt as a basic form [11]. According to Husserl the experience, sensation and perceptions of a life form in a day-to-day environment is a horizon of life, which is concrete as well as local, and it is very different from the world horizon which follows a set of pure scientific or mathematical principles. Interestingly, the existentialism of Husserl has similar perspectives as compared to Platonism and the noumenal (i.e., unknown realm of freedom) elements of Kant [11]. The use of absolute rationalism to explain the world as a phenomenon (local events) fails to explain many day-to-day observations in the world horizon. For example, the statement derived from absolute realism saying that "water boils at $100{ }^{\circ} \mathrm{C}$ temperature" is not correct in the world horizon because it depends on the location of water in space. Thus, the existential forms, time and position, are important parameters to determine the locally observable or sensually measurable properties of the forms. It is important to note that Jean-Paul Sartre provided a convincing argument in favor of the existentialism of Husserl, Heidegger and Kierkegaard [11].

The philosophical approaches of Spinoza regarding existentialism have few similarities and some differences with respect to the philosophy of mathematician Leibniz. The existentialism of Spinoza has a twofold meaning. Spinoza specifically stressed on the principle of ground-consequent, and in this relation, the causation is identical [12]. As a result, all determined parameters become transitory and a series of cause-effect relational chains are formed where all consequences can be derived from a set of primitive causes. It is important to note here that the Aristotelian method of logical deductions sometimes fails to achieve clear uniformity if the system under investigation is complex [12]. On the other hand, Leibniz philosophized that all matter can be properly understood through abstract mathematical entities embodying the properties of corresponding matter. According to Leibniz, the characteristic feature of every substance is unity, and that unity is not conceivable just by the appearance of the substance in its forms and an activity is required [12]. It is important to note that the monadism of Leibniz may have some Platonic elements, 
and the concept of continuous evolution is embodied within the mathematical philosophy of Leibniz.

\subsection{Dualism}

The mind-body relation and dualism are intricately linked where a body is considered to be the material in a form. The notion of Hegelian idealism is contrary to the neomaterialism and the idealism attracted support from Bradley, Sir Eddington and Sir Jeans in the field of science [13]. Apart from the Thomistic approach, the unambiguous dualism enables the investigation of nature by removing the forms and species, while formulating a set of scientific principles exposing the laws of nature [14]. Fortunately, mathematics always, from antiquity to today, plays an unavoidable and extremely crucial role in the process. The dualism transformed the Aristotelian understanding of matter or materials and various other approaches are revived, such as atomism, skepticism and nominalism [14]. As a result, nature, matter and forms appear highly mathematical, exposing inherent natural laws, principles and their accurate interpretations [14]. This effectively gives way to the process of mathematical abstractions to understand nature as a set of purely mathematical principles.

\subsection{Abstractionism}

In the field of mathematical philosophy (and philosophy of mathematics), it is a well-accepted concept that humans make references to and deal with a large set of abstract objects or entities in everyday life [15]. The ontological and epistemological misunderstandings arise if one argues that abstract objects are not located in physical space. However, in this case the basic questions are: What is a space? Is it not the fact that the mathematical concept of space is also an abstract entity? According to Boolos and Gödel, abstract objects are understood through perceptual contacts in the everyday life of human beings [15]. For example, human or even some other life forms in nature count by recognizing numbers. Note that numbers are abstract (invisible) Platonic entities and humans are making perceptual contacts with them cognitively to make everyday life fruitful, indicating that such abstract mathematical objects exist in the universe. It is mentioned earlier that the perceptual understanding and measurement of contacts to living environment is highly limited in materialistic senses. Frege suggested that the meaning of abstract identity statements should be understood through finding out a suitable equivalence relation between them. Suppose $S, T$ are two abstract objects (while retaining the possibility of isomorphism). The function form $f(a)$ can induce an equivalence relation $\Phi a b$ as given in the following functional expression [15]:

$$
\Phi x y \equiv[f(x)=f(y)]
$$

This invites the problem for finding an equivalence relation, which can be hard. Moreover, if such equivalence relation does not exist, then what would be the solution? The Platonic Neo-Frege theory illustrates that by employing abstraction principles, one can make perceptual contact with abstract objects, which does not necessarily need to maintain reductionism because abstract mathematical realm is in pure state.

\section{Random Choices for Existence}

The constructive mathematical as well as philosophical analysis of elemental life deals with the life forms of elements in a natural environment. In human society, an elemental life form is an individual in space-time having a mind-body relation in a birth-death process where the society is viewed as an ensemble of structure(s) composed of elements and their mutual interactions. This perspective of elemental life forms and society allows the evolution of societal and cultural structures over time, although no guarantee is made to achieve optimality or perfection. As a result, the corresponding evolutions of elemental life forms appear to be a continuous process, although the changes are unnoticed in smaller time-scales (in local-time horizon). Note that the cosmological time-scale of the universe is extremely large as compared to the local-time horizon of the elemental life forms. 
The complex behaviors of elemental life forms as the social interactive elements are theorized in view of social choice theory and game theory affecting economic structures [16]. In this paper, we take a different discourse, and we will try to constructively formulate a mathematically consistent model of an elemental life form and its dynamics in the birthdeath process in cosmological space-time (i.e., universe-time horizon). Our effort in this paper will be to offer a constructive treatment to elemental life forms in view of mathematics and philosophy, which is coherent to the materialistic biophysical understandings and experiences. The basis of a materialistic element is its biochemical structures and their mutual reaction pathways determining the dynamics of life of an element. The materialistic formation of an element begins when two genetic materials combine in a suitable growth environment, which we call as a birth of an element in environment or nature. As a result, the dynamics and properties of the elemental life forms are pre-determined by the genetic materials in a combination. However, the limitation of this viewpoint becomes considerable due to the fact that the life forms and natural environments mutually influence each other in bidirectional manner $[17,18]$. Hence, the dynamics of elemental life and its evolution are not purely pre-determined in a constantly changing environment and evolutionary discourse appears over time. As a result, the evolution of elemental life forms and the dynamics are not deterministic in full and appear to be probabilistic in nature based on some purely random choices satisfying the instantaneous requirements for existence in an environment. This can be considered as a process of random choices for instantaneous existences. This motivates to search a new approach and perspective to understand and analyze the elemental life forms by combining both mathematical and philosophical constructivism. First, we define a set of concepts in view of mathematical philosophy as follows:

Definition (Birth-death process): LetU be the entire universe of existence. A birth-death process of an existential life form (as an element $p$ ) in the cosmological time-scale is the local dynamics of a continuous function $f_{p}(t)$ between the two fixed points in the continuous interval $\left[t_{B}(p), t_{D}(p)\right]$ for the corresponding element. The dynamics of $f_{p}\left(\left[t_{B}(p), t_{D}(p)\right]\right)$ are in continuum, and the discourse is determined by a sequence of probabilistic events attached to $p$ in $U$.

Every existential life form finitely interacts to the environment in local time-scale in the universe, and the number of elements in the environment are also finite for the life form for interaction within $\left[t_{B}(p), t_{D}(p)\right]$. As a result, an existential life form makes a set of finite choices in the environment during the process of interaction, which is defined as follows:

Definition (Selectable finite choices): For all existential element $p$ there is a finite $E \subset U$ forming an environment of $p$ from which the existential element makes finite choices in $\left[t_{B}(p), t_{D}(p)\right]$. The choices made by the existential life form are the selection set $E_{p} \subset E$ of that element.

The choices made by an existential life form out of selection set are probabilistic as determined by the element, and it affects the dynamics of the birth-death process and a set of such processes form a homotopy:

Definition (Homotopy forms): $A$ set $\left\{f_{p}\left(\left[t_{B}(p), t_{D}(p)\right]\right): p \in U\right\}$ forms homotopy if $\forall p, f_{p}\left(t_{B}(p)\right)<f_{p}\left(t_{D}(p)\right)$ in local time-horizon and $\forall p, q \in U,\left[f_{p}\left(t_{B}(p)\right) \approx f_{q}\left(t_{B}(q)\right)\right] \wedge$ $\left[f_{p}\left(t_{D}(p)\right) \approx f_{q}\left(t_{D}(q)\right)\right]$ in the cosmological time-horizon which is a half-open infinite space of Sorgenfrey line.

The cosmological time-horizon is much larger than the local time-horizon of a set of birth-death processes resulting into the formation of the homotopy in the cosmological time horizon. 


\subsection{Existential Formation of Elements: A Probability Chain}

In view of mathematical constructivism, an element comes into a materialistic existence by following two randomized processes producing a set of probabilistic outcomes of events. Let a set of elements in a dynamic natural environment at time $t$ be denoted by $X(t) \subset U$. The first random process in $X(t)$ is denoted as $P_{M}(p, r)$, where $\exists p \exists r \in X(t)$ are the elements. The process $P_{M}(p, r)$ signifies the probability of the pairing of respective elements in the presence of selectable finite choices. The second random process is denoted as $Q(\{p, r\}, q)$ signifying the probability of forming an element $\exists q \in X\left(t_{2}\right)$ if and only if $P_{M}(p, r)$ and $P_{M}(r, p)$ are successful birth-death processes at $t_{1}<t_{2}$. We will logically establish later that $P_{M}(p, r)$ and $P_{M}(r, p)$ need not be always exactly equal. Suppose $t_{\alpha} \in\left(t_{1}, t_{2}\right)$ is an uncertain time instant (i.e., not predetermined) within the open interval. Let us algebraically denote the formation of element $q \in X\left(t_{2}\right)$ as $P_{B}(q \mid\{p, r\}) \mid t_{2}$. Accordingly, for clarity, let us denote $P_{M}(p, r)\left|t_{1}, P_{M}(r, p)\right| t_{1}$ and $Q(\{p, r\}, q) \mid t_{\alpha}$ signifying that these probabilistic events are measured at specific time instants in local as well as cosmological time-scale horizons as indicated. Note that the local time-scale horizon is a continuum, and it is an uncountable subset of cosmological time-scale horizon. The probability of combined birth-death processes generating an element are given in the following equation computed under an abstract algebraic operation (i.e., not necessarily multiplication at this point; detailed analysis is presented later in this section considering deterministic algebraic operations):

$$
P_{B}(q \mid\{p, r\})\left|t_{2}=\left[\left(P_{M}(p, r) \mid t_{1}\right) \cdot\left(P_{M}(r, p) \mid t_{1}\right)\right] \cdot Q(\{p, r\}, q)\right| t_{\alpha} .
$$

It is important to note the following properties of these two processes while in a combination exerting mutual influences satisfying the stability of structures and evolution:

$$
\begin{aligned}
& {\left[\left(P_{M}(p, r) \mid t_{1}\right) \neq\left(P_{M}(r, p) \mid t_{1}\right)\right] \Rightarrow\left[\left(P_{M}(p, r) \mid t_{1}\right)<1\right] \wedge\left[\left(P_{M}(r, p) \mid t_{1}\right)<1\right],} \\
& {\left[\left(P_{M}(p, r) \mid t_{1}\right) \cdot\left(P_{M}(r, p) \mid t_{1}\right)\right]=\left[\left(P_{M}(r, p) \mid t_{1}\right) \cdot\left(P_{M}(p, r) \mid t_{1}\right)\right],} \\
& {\left[\left(P_{M}(p, r) \mid t_{1}\right) \cdot\left(P_{M}(r, p) \mid t_{1}\right)\right] \cdot\left(Q(\{p, r\}, q) \mid t_{\alpha}\right) \neq} \\
& \left(P_{M}(p, r) \mid t_{1}\right) \cdot\left[\left(P_{M}(r, p) \mid t_{1}\right) \cdot\left(Q(\{p, r\}, q) \mid t_{\alpha}\right)\right] .
\end{aligned}
$$

From the aforesaid properties, we can conclude that the random birth-death processes of pairing of elements are in a commutative relation; however, the combined processes for the generation of an element are not associative in nature (i.e., the abstract algebraic operation is commutative but not associative). It indicates that the operation generating the relation is at least not an algebraic division because, in that case, commutativity will not be valid (because division is not commutative). On the contrary, if the commutativity is valid with respect to the algebraic division operation, then it results in the conclusion that $\left(P_{M}(p, r) \mid t_{1}\right)=\left(P_{M}(r, p) \mid t_{1}\right)=1$. However, in this case, the associativity will be achieved violating the principle in the local time-scale horizon of birth-death processes. Moreover, the non-associativity of the algebraic operation indicates that it is not an addition or a multiplication operation. Thus, the abstract algebraic operation generating such relation (commutative and non-associative) is sensitive to instantaneous time of measurement and cannot guarantee associativity under future projection in time, where the time is considered as a half-open infinite space of the Sorgenfrey line. In other words, the proposed construction includes the uncertainty in the time-scale horizon about the formation of events as presented in the following equation, considering that the abstract algebraic operation is a multiplication:

$$
\begin{aligned}
& {\left[\left(P_{M}(p, r) \mid t_{1}\right)=\left(P_{M}(r, p) \mid t_{1}\right)=1\right] \Rightarrow} \\
& {\left[\left(P_{M}(p, r) \mid t_{1}\right) \cdot\left(P_{M}(r, p) \mid t_{1}\right)\right] \cdot\left(Q(\{p, r\}, q) \mid t_{\alpha}\right)=} \\
& \left(P_{M}(p, r) \mid t_{1}\right) \cdot\left[\left(P_{M}(r, p) \mid t_{1}\right) \cdot\left(Q(\{p, r\}, q) \mid t_{\alpha}\right)\right] .
\end{aligned}
$$

It is important to note that if the abstract algebraic operation is multiplication and the probabilities $P_{M}(p, r)$ and $P_{M}(r, p)$ are each unity signifying certainty, then Equation (2) 
generates the absolute probability of the generation of a new element and Equation (3) becomes transformed into a commutative as well as associative under such certainty of pairing of elements. However, even in this case there is no guarantee that $\left(Q(\{p, r\}, q) \mid t_{\alpha}\right)=1$ will be achieved in Equation (4). Furthermore, Equation (2) exposes the fact that if $\left[\left(P_{M}(p, r) \mid t_{1}\right)<1\right] \wedge\left[\left(P_{M}(r, p) \mid t_{1}\right)<1\right]$, then $P_{B}(q \mid\{p, r\}) \mid t_{2}$ cannot determine with absolute certainty whether a new element can be formed or not in the universe although commutativity is preserved (In this case associativity is not preserved in projection at different instants of time).

Interestingly, it is well known that the stable existence of $q \in X\left(t_{2}\right)$ is a highly probabilistic event due to the presence of several natural environmental factors affecting the existentialism of materials. We need to emphasize that although $Q(\{p, r\}, q) \mid t_{\alpha}$ generates a probabilistic event, the observation $H(q)$ of event of formative existence of an element $q \in X\left(t_{2}\right)$ is a discrete function, as follows:

$$
\begin{aligned}
& \left(Q(\{p, r\}, q) \mid t_{\alpha}\right) \in[0,1], \\
& H(q) \in\{0,1\} .
\end{aligned}
$$

Note that the theory of natural selection approximately determines the formation of the material form of an element and its evolving existence in a natural environment. It is relatively straightforward to observe that the theory of natural selection is maintained by the proposed constructive mathematical formalism.

\subsection{Relations to Empty, Entire and Observations}

One can analyze two cases of existential formation of the elemental life forms in a probability chain. The proposed analytical discourse combines the ingredients of mathematics, philosophy and social sciences. Let us first consider that the relation of an element with the empty, $\phi$. Let us consider the related algebraic structure which can be denoted as $P_{M}(p, \phi) \mid t_{+\infty}$, where $t_{+\infty} \in\left[t_{1},+\infty\right]$. If we analyze the extremely limiting but stable choice of the element $p \in X(t)$, then we can deterministically conclude that $\left[\left(P_{M}(p, \phi) \mid t_{+\infty}\right)=1\right] \wedge\left[\left(P_{M}(\phi, p) \mid t_{+\infty}\right)=1\right]=1$. This directly results in the following logical conclusion in a birth-death process due to the degeneration of probabilistic measurement into the binary-valued determinism:

$$
\left(P_{B}(q \mid\{p, \phi\}) \mid t_{2}\right) \vee H(q)=0
$$

On the other extreme end, let us consider the structure $P_{M}\left(p, X\left(t=t_{n}\right)\right) \mid t_{+\infty}$ encompassing the whole. Clearly, this structure is not stably sustainable due to scale, and as a result, we can infer an equivalence relation between the empty and the whole as follows, following the degenerative probabilistic measurement into binary-valued determinism:

$$
\left(P_{B}\left(q \mid\left\{p, X\left(t_{n}\right)\right\}\right) \mid t_{2}\right) \vee H(q)=\left(P_{B}(q \mid\{p, \phi\}) \mid t_{2}\right) \vee H(q) .
$$

Interestingly, the probabilistic outcome of both opposite extremes are the same, and the proposed construction also represents that once the probabilistic existential events are successfully chosen randomly (i.e., not 0 ) in time, then the corresponding observations are determined. If the probabilistic existential conditions of an element $q$ are stable and satisfied with high probability, then $H\left(q \in X\left(t_{2}\right)\right)=1$, reaching certainty. Otherwise, the model predicts that $H\left(q \in X\left(t_{2}\right)\right)=0$ is a natural consequence in the natural environment. Furthermore, it exposes two different pathways of an elemental life form to evolve over time by following the homotopy theory of algebraic topology [19]. The topological homotopy theory appears to be a close fit to establish and explain the dynamics of elemental life forms in the cosmological time-scale of the universe in terms of constructive mathematical as well as philosophical principles. 


\section{Homotopic Existential Analysis of Elements}

In the homotopic analysis of the existence of elements, we will consider two different time-scales: local and cosmological. The local time-scale is a subset of cosmological (universal) time-scale, where both are continuous in nature. We represent cosmological time scale as $T_{C}$, and we consider that $T_{C} \cong R_{+\infty}$, where $R_{+\infty}=[0,+\infty]$, is the subset of extended real numbers. Evidently, the local time-scales of an elemental form and its evolution is much shorter than the cosmological time-scale, where the cosmological timescale is equivalent to a half-open (lower-limit) infinitary Sorgenfrey positive array [20]. Note that, in this case, the Minkowski space-time structure of topological space is decomposed and the time-like space is considered in our constructions and analysis. This observation leads to a set of interesting analytical insights in mathematical philosophy. In this section the emphasis is made on the homotopic analysis, and it omits the specified time instants within the local time-scale horizon for easy representation.

\subsection{Homotopic Existence for $H(q)=0$}

The application of homotopy theory in analyzing the existential conditions of an element form for $H(q)=0$ reveals a new philosophical inroad of understanding. In view of homotopy theory, we can derive two different existential conditions of an element $q \in X(t)$ by considering the birth-death process, where $t_{B}(q), t_{D}(q)$ represents the time of birth and death of the corresponding element in the local time-scale. These conditions are presented as follows:

$$
\begin{aligned}
& \forall p \forall r \exists q \in X(t), \\
& {[Q(\{p, r\}, q)=0] \Rightarrow[H(q)=0],} \\
& {[H(q)=0] \wedge[Q(\{p, r\}, q) \in[0,1)] \Rightarrow\left[t_{D}(q) \approx t_{B}(q)\right] .}
\end{aligned}
$$

Hence, according to homotopy theory, this can be viewed as a formation of a trivial fundamental group denoted by $\pi_{1}(X(t), q)$ such that the homotopy class $[q]$ becomes a left as well as a right identity as a single element in $\pi_{1}(X(t), q)$ due to the fact that $\left(\left[t_{D}(q)-t_{B}(q)\right] / T_{C}\right) \rightarrow 0$. In other words, $[q]$ is the nullhomotopy class representing $\pi_{1}(X(t), q)$ of a birth-death process in the cosmological time-scale horizon. This mathematical constructive approach validates the associated philosophical understanding of elemental life in a birth-death process in the observable local time-scale and its duality in the universe in the cosmological time horizon.

\subsection{Homotopic Existence for $H(q)=1$}

This is another extreme where the probabilistic outcomes of events are deterministically observable in a natural environment. It is relatively straightforward to observe that, in this case, the following properties are maintained in the universe:

$$
\begin{aligned}
& \varepsilon(q)=t_{D}(q)-t_{B}(q)>0, \\
& \exists F \exists K \in R_{+\infty}, F<<+\infty, K<<1, \\
& \varepsilon(q) \in(0, F], \\
& \frac{F}{T_{C}} \in(0, K) .
\end{aligned}
$$

Thus, one can infer from the materialistic as well as mathematical philosophy standpoints that the birth-death process of an observable element is deterministic with the corresponding two fixed points: $t_{B}(),. t_{D}($.$) . As a result, an n$-dimensional path-homotopy structure is formed in local time-scale horizon within the cosmological time-scale hori- 
zon for all existential and observable elements of life forms, which can be represented as follows:

$$
\begin{aligned}
& \left|X\left(t=t_{m}\right)\right|=N(m) \in Z^{+}, N(m) \in(0,+\infty), \\
& \forall p \in X\left(t \in\left[0, t_{m}\right)\right), f_{p}:[0,1] \rightarrow\left(\left[t_{B}(p), t_{D}(p)\right] \subset T_{C}\right) \times\left(S^{n-1} \subset R_{+\infty}^{n-1}\right), \\
& \forall p \forall q \in X\left(t \in\left[0, t_{m}\right)\right),\left|\left[\varepsilon(p) / T_{C}\right]-\left[\varepsilon(q) / T_{C}\right]\right| \rightarrow 0, \\
& \left(\left(\left|f_{p}(0)-f_{q}(0)\right| / T_{C}\right) \approx 0\right) \wedge\left(\left(\left|f_{p}(1)-f_{q}(1)\right| / T_{C}\right) \approx 0\right) \Rightarrow \\
& {\left[f_{p}(0) \approx f_{q}(0)\right] \wedge\left[f_{p}(1) \approx f_{q}(1)\right] .}
\end{aligned}
$$

Note that the path-homotopy expression is considering a generalized $n$-dimensional world horizon and an $n$-dimensional local horizon of perception. If we consider perceivable space-time as $n=4$, then it is transformed into a four-dimensional perceivable space-time environment of the existential life forms. The observation $\varepsilon(p) \approx \varepsilon(q)$ derived from the above equations may appear surprising, but it is valid because it is always true that $\forall p \in X(t), 0<\left(\varepsilon(p) / T_{C}\right)<<1$. It is important to note that each $f_{p}($.$) is continuous$ representing dynamics of elemental life form of each discrete element in a local time-scale, and the path-homotopy functions of all elements are between the two fixed points in the cosmological time-scale of universe. However, this constructive approach does not violate another important material property, such as the actual local time-span of an elemental form in a homotopy of birth-death processes. If $L_{p}$ represents the local time-span of an elemental life form in a path-homotopy, then one can compute $L_{p}$ as follows by using line integral in $n$-dimensional space:

$$
\begin{aligned}
& L_{p}=\int_{l} f_{p}(t) d t \\
& \left(L_{p} / T_{C}\right) \in(0, K) .
\end{aligned}
$$

The line integral is computable because path-homotopic functions are continuous. However, one can conclude further that for any two elements $\exists p \exists q \in X(t)$, the values of $L_{p}, L_{q}$ may be equal or not equal depending on the individual elemental dynamics in the local time-scale. As a result, one can infer that $\forall p \forall q \in X(t), \exists a \in R_{+\infty},\left(L_{p} / L_{q}\right) \in$ $(0, a)$, where $0<a<<+\infty$. This validates that the proposed constructive mathematical formalism maintains the corresponding philosophical interpretations of elemental life forms in local time-scale and also in the cosmological time-scale.

\subsection{Consistency of Transitions}

In the previous sections, we have presented two different abstractionisms, namely, probabilistic existentialism and homotopic existentialism. These two abstract forms are mutually consistent and there is no logical conflict between the two. In this section, we present a brief analytical outline illustrating that consistency of an equivalence class $S(t)=\{p, q, r\}$ is independent of nature of existentialism, where $S(t) \subset X(t)$. Moreover, the consistency of $S(t)$ is maintained under observable transitions in the cosmological timescale horizon $T_{C}$. Suppose we consider a path-homotopy of elements in $S(t)$ in cosmological time-scale horizon $T_{C}$ where the path-homotopy is maintained within local time-scale horizon $\left[t_{a}, t_{b}\right]$ such that $0<\left(\left(t_{b}-t_{a}\right) / T_{C}\right)<<1$. It indicates that from the observational point of view one can conclude that $\forall t \in\left[t_{a}, t_{b}\right], \exists x \in S(t), H(x)=1$. Note that this is a highly relaxed version considering individual elements at any instant of time within the path-homotopic time interval in local time-scale horizon. A stronger form of observation can be abstracted as: $\forall t \in\left[t_{a}, t_{b}\right], H(p) \vee H(q) \vee H(r)=1$. An equivalence class $S(t)$ is called stable at a time $t \in\left[t_{a}, t_{b}\right]$ if, and only if, $\exists t \in\left[t_{a}, t_{b}\right], H(p) \wedge H(q) \wedge H(r)=1$. It is relatively easy to infer that $\forall t \in\left[0, t_{a}\right) \subset T_{C}, \forall t \in\left(t_{b},+\infty\right] \subset T_{C}, H(p) \vee H(q) \vee H(r)=0$. Thus, there is a 0-0 existential transition in the cosmological time-scale horizon for every element in $X(t)$ except the path-homotopy interval, which is in a local time-scale horizon. In other words, the existential life forms are in an equivalent class of the 0-0 state everywhere outside of the corresponding local path-homotopy interval. Moreover, it indicates that there is an extended interval $t_{a e}<t_{a}, t_{b e}>t_{b},\left[t_{a e}, t_{b e}\right]$ where almost every existential life 
form in universe $U$ will preserve the $0-0$ transition in the cosmological time-scale horizon, which is in line with the theory of evolution and disappearance of species in nature.

\section{Conclusions}

It is generally an accepted theory in evolutionary biology and social choice theory that life forms in nature follow the dynamic trajectories highly influenced by the probabilities of various events in nature. However, in the field of philosophy, it raises debate mainly between Platonic and realism schools of thoughts, which is further detailed towards resolution by neo-realism and New-logic. The proposed structure in light of mathematical philosophy applies the probability theory and predicate logic to illustrate that it is possible to establish a coherent structure of understanding. Moreover, the applications of topological homotopy theory nearly accurately encapsulate the conceptual and constructive understanding of existential life forms in nature without any observable contradictions. The cosmological time-scale representing the world or universal horizon plays an important role to formulate homotopic structures of dynamics of existential life forms in nature. The constructive mathematical treatment proposed in this paper enables us to coherently amalgam the structural notions of the local time-scale horizon and cosmological time-scale horizon of space-time through the lenses of mathematical philosophy.

Funding: The research is funded by Gyeongsang National University, Jinju, Korea.

Data Availability Statement: Not applicable.

Acknowledgments: The author would like to thank the anonymous reviewers and editors for their valuable comments and suggestions.

Conflicts of Interest: The author declares no conflict of interest.

\section{References}

1. Parsons, C. Informal axiomatization, formalization and the concept of truth. Synthese 1974, 27, 27-47. [CrossRef]

2. Heis, J. "Critical philosophy begins at the very point where logistic leaves off": Cassirer's response to Frege and Russell. Perspect. Sci. 2010, 18, 383-408. [CrossRef]

3. Anderson, A. Alonzo Church's contributions to philosophy and intensional logic. Bull. Symb. Logic 1998, 4, 129-171. [CrossRef]

4. Netz, R. The Pythagoreans. In Mathematics and the Divine: A Historical Study; Elsevier: Amsterdam, The Netherlands, 2005; Chapter 3; pp. 79-97.

5. Igamberdiev, A.U. Time and life in the relational universe: Prolegomena to an integral paradigm of natural philosophy. Philosophies 2018, 3, 30. [CrossRef]

6. Bekenstein, J. Universal bound on the entropy to energy ratio for bounded systems. Phys. Rev. D. 1981, 23, 287-298. [CrossRef]

7. Thagard, P. Naturalizing logic: How knowledge of mechanisms enhances inductive inference. Philosophies 2021, 6, 52. [CrossRef]

8. Parsons, C. Infinity and Kant's conception of the "Possibility of Experience". Philos. Rev. 1964, 73, 182-197. [CrossRef]

9. Russell, L.J. Some problems in the philosophy of Leibniz. In Meeting of the Aristotelian Society; Oxford Academy: Oxford, UK, 1923; Chapter XII.

10. Santos, T.J. On a multiplicity: Deconstructing Cartesian dualism using mathematical tools in performance. Liminal. J. Perform. Stud. 2014, 10, 1-28.

11. Wild, J. Existentialism as a philosophy. J. Philos. 1960, 57, 45-62. [CrossRef]

12. Hicks, G.D. The "Modes" of Spinoza and the "Monads" of Leibniz. In Proceedings of the Aristotelian Society; Oxford Academy: Oxford, UK, 1918; Volume 18, pp. 329-362.

13. Forster, E. Twenty-five years of philosophy. Nature 1935, 136, 332. [CrossRef]

14. Balz, A.G.A. Dualism and early modern philosophy. II. J. Philos. Psychol. Sci. Methods 1918, 15, 225-241. [CrossRef]

15. Heck, R.G. The existence (and non-existence) of abstract objects. In Abstractionism: Essays in Philosophy of Mathematics; Oxford University Press: Oxford, UK, 2016; Chapter 3; pp. 50-78.

16. Maschler, M.; Solan, E.; Zamir, S. Social choice. In Game Theory; Cambridge University Press: Cambridge, UK, 2013 ; pp. 853-883.

17. Rozzi, R. The reciprocal links between evolutionary-ecological sciences and environmental ethics. BioScience 1999, 49, 911-921. [CrossRef]

18. Corris, A. Defining the environment in organism-environment systems. Front. Psychol. 2020, 11, 1-13. [CrossRef] [PubMed]

19. Jardine, J. Local Homotopy Theory; Springer: New York, NY, USA, 2015; ISBN 978-1-4939-2300-7.

20. Douwen, E.K.V.; Pfeffer, W.F. Some Properties of the Sorgenfrey Line and Related Spaces. Pac. J. Math. 1979, 81, $371-377$. [CrossRef] 